\title{
Rational Design of Lanthanoid Single-Ion Magnets: Predictive Power of the Theoretical Models
}

José J. Baldoví,a Yan Duan, a Roser Morales,b Alejandro Gaita-Ariño, a Eliseo Ruiz *, b and Eugenio Coronado,*, a

a Instituto de Ciencia Molecular (ICMol), Universidad de Valencia, C/Catedrático José Beltrán 2, E-46980 Paterna, Spain.

b Departament de Química Inorgànica and Institut de Recerca de Química Teòrica i Computacional, Universitat de Barcelona, Diagonal 645, E-08028 Barcelona, Spain.

KEYWORDS single-ion magnet, ab initio, effective electrostatic model, lanthanides, oxydiacetate.

Supporting Information Placeholder

ABSTRACT: We report two new single-ion magnets (SIMs) of a family of oxydiacetate lanthanide complexes with D-3 symmetry to test the predictive capabilities of complete active space ab initio methods (CASSCF and CASPT2) and the semiempirical radial effective charge (REC) model. Comparison of the theoretical predictions of the energy levels, wave functions and magnetic properties with detailed spectroscopic and magnetic characterisation is used to critically discuss the limitations of these theoretical approaches. The need for spectroscopic information for a reliable description of the properties of lanthanide SIMs is emphasised.

\section{Introduction}

The magnetism of lanthanides has intrigued researchers for decades. This interest increased with the discoveries, first in solid state and then in the field of molecular magnetism, of $4 f$ ion mononuclear complexes presenting slow relaxation of the magnetization, i.e. with single-molecule magnet (SMM) behaviour.1,2 In the past decade, the impact of mononuclear SMMs, also known as single-ion magnets (SIMs), has dramatically increased.3,4,5 This kind of molecular compounds exhibits attractive physical properties such as magnetic hysteresis at low temperatures and quantum phenomena.6,7

In contrast to the first generation of SMMs discovered in the 90's,8 magnetic clusters whose properties rely on the ability of exchange interactions to stabilize anisotropic high spin states, the magnetic and quantum properties of mononuclear SMMs, which are the second generation of SMMs, depend primarily on the anisotropy of a single ion. This in turn results from a strong spinorbit coupling and an adequate ligand field. The magnetic and spectroscopic properties of lanthanide complexes is commonly analysed using crystal field theory, which unfortunately requires the determination of a large number of crystal field parameters (CFPs), usually resulting in overparameterisation. Thus, modelling the properties of $f$-element complexes remains an open problem. The first approach to deal with this, which is broadly used by spectroscopists, is the extraction of phenomenological CFPs from a direct fit of UV/visible spectroscopic experimental data.9 A full experimental determination of the lying magnetic levels can also be obtained from techniques like far infrared (FIR), inelastic neutron scattering spectra (INS) or magnetic circular dichroism (MCD) spectroscopy. 10,11 Experimental advances in the field aiming to address this issue include the high resolution luminescence spectroscopic method, which has been applied to determine the fine electronic structures of lanthanide complexes.12,13 From the computational point of view, a substantial advance is the CONDON program, which uses the full Hamiltonian and determines the phenomenological CFPs from a fitting of the magnetic susceptibility data and/or energy levels. 14,15 Unfortunately, CONDON does not relieve the overparameterisation problem and for many systems there are no available spectroscopic data, so it is very common that independent theoretical techniques are required to estimate an initial set of CFPs.

A different strategy involves the direct calculation of CFPs and all the observables using the real structure of the compounds. The simplest procedure is based on the point charge electrostatic model (PCEM), 16 subsequently improved by several semiempirical models.17,18,19,20,21,22 These models, while very useful for quick predictions of the properties and for obtaining an initial set of CFPs, also need parameterisation to take into account covalency to provide a more realistic description of the observed properties of the system. A more expensive approach involves the calculation of the energy levels using 
electronic structure $a b$ initio methods. In general, this last approach has practically been the default option for the theoretical characterisation of SIMs as first principles methods are expected to be more exact compared with the more intuitive electrostatic methods. 23, 24, 25 However, when spectroscopic techniques are used to determine the energy levels, evidences of important deviations between the theoretical predictions, obtained by these methods with the experiment have been accumulating recently.10,11,26 While both electrostatic and $a b$ initio methods are nowadays considered standard tools in the field, there is a lack of benchmark works to critically compare their performances, study their limitations and analyse their pros and cons. To advance in this direction we should reverse the methodology used so far. Thus, instead of using the theory as a substitute for spectroscopic information, one should take a system where the energy levels have already been measured by high-quality spectroscopic experiments, blindly "predict" the energy level scheme using the two kinds of theoretical models and, finally, compare the experiment with the theoretical predictions. An interesting study along this direction have been performed using SQUID magnetometry and INS measurements, 11 as well as the work of Marx et al.10 where far IR and INS spectroscopies were employed. Nevertheless, these works did not deeply analyse the reported divergences between theory and experiment. The only effort to try to approach the experiment has been to introduce scaling factors in these models without any proper justification.

In this work we have used the series $\mathrm{Na} 5\left[\operatorname{Ln}(\mathrm{oda})_{3}\right]\left(\mathrm{H}_{2} \mathrm{O}\right)_{6}(\mathrm{BF} 4)_{2}$, where $\mathrm{Ln}=\mathrm{Dy}_{3}+, \mathrm{Er}_{3}+$ and oda $=$ oxydiacetate $\left(\mathrm{C}_{4} \mathrm{H}_{4} \mathrm{O}_{52}-\right)$, in short DyODA and ErODA respectively, as benchmark systems to test and compare the capabilities of these two kind of theoretical models. We selected these two isostructural compounds because of the availability of a combination of spectroscopic measurements and the fact that their calculated ground doublet wave functions suggested they could exhibit SMM behaviour.27,28 In the first part, we will perform an experimental magnetic characterisation of the compounds, checking for SMM behaviour. Then, in the second part we will apply different levels of $a b$ initio and electrostatic calculations to discuss their ability to predict the experimental spectroscopic and magnetic information.

\section{Experimental}

The syntheses of DyODA and ErODA were performed by following a previously described procedure. 29 All reagents were commercially purchased and used without any further purification.
DyCl $3 \cdot 6 \mathrm{H}_{2} \mathrm{O}$ and $\mathrm{ErCl}_{3} \cdot 6 \mathrm{H}_{2} \mathrm{O}$ are highly hygroscopic and were stored in a desiccator. After the syntheses, pure block-shaped colourless crystals of DyODA and pure block-shaped pink crystals of ErODA were obtained by recrystallization of the crude sample in a minimum amount of milli-Q water.

The compounds were characterised by FT-IR spectroscopy (figures S1 and S2), X-ray power diffraction and single-crystal X-ray diffraction. The unit cell parameters are coincident with the ones reported previously 29 (see figures S3 and S4).

IR spectra were recorded on an FT-IR Nicolet 5700 spectrometer in the 2000-400 cm-1 range using powdered crystals in $\mathrm{KBr}$ pellets. For DyODA (Fig. S1), FT-IR spectroscopy data in the range 2000-400 cm-1 (KBr pellet): 1622(s), 1459(s), 1423(s), 1362(s), 1317(s), 1247(m), 1126(s), 1084(s), 1047(s), 959(m), 935(s), 709(m), 604(w), 571(m), 534(w), 522(s). For ErODA (fig. S2), FT-IR spectroscopy data in the range 2000-400 cm-1 (KBr pellet): $1620(\mathrm{~s})$, 1427(s), 1366(s), 1320(s), 1124(m), 1050(s), 958(m), 935(s), 710(m), 609(w), 571(m), 523(w).

Polycrystalline samples of both derivatives were lightly ground in an agate mortar and pestle and filled into $0.7 \mathrm{~mm}$ borosilicate capillaries prior to being mounted and aligned on a Empyrean PANanalytical powder diffractometer, using $\mathrm{Cu} \mathrm{K \alpha}$ radiation $(\lambda=1.54056 \AA)$. For each sample, three repeated measurements were collected at room temperature $\left(2 \theta=2-40^{\circ}\right)$ and merged in a single diffractogram. For both compounds, powder-XRD verified that the bulk samples consist of a single phase, which corresponds to the crystal structures (as shown in figs. S3 and S4).

Magnetic susceptibility, $\chi m$, data were measured between 2 and $300 \mathrm{~K}$ with a commercial magnetometer equipped with a SQUID sensor and a commercial physical properties measurement system (PPMS). The diamagnetic contributions to the susceptibility were corrected using Pascal's constant tables. Direct current (dc) data were collected with an applied field of 1000 Oe and also without field. Alternate current (ac) data were collected in the range 2-10 $\mathrm{K}$ with an applied dc field of $1000 \mathrm{Oe}$ at different frequencies in the range $1500-10000 \mathrm{~Hz}$.

For the theoretical characterization of the spectroscopic and magnetic properties, we use two well-established theoretical approaches: Complete Active Space $a b$ initio calculations 30 and the semiempirical electrostatic method based on Radial Effective Charge (REC) model 22.

For the electronic structure $a b$ initio study, we performed post Hartree-Fock calculations based on the relativistic quantum chemistry method CASSCF+RASSI implemented in the MOLCAS 8.0 software package for DyODA and ErODA.31 CASSCF calculations were performed for three different 
multiplicities (sextet, quartet and doublet) for the Dy complex, and two multiplicities (quartet and doublet) for the Er complex. We included 21, 128 and 98 states for the sextet, quartet and doublet calculations of the Dy complex. While for the Er derivative we included 35 and 112 states for the quartet and doublet calculations. In the case of the CASPT2 calculations of the ground state multiplicities, we included 21 states for the sextet of Dy and 13 states for the quartet of Er. The effect of spin-orbit coupling on the basis of the converged wave functions obtained in the previous step (CASSCF or CASPT2) is included by the Restricted Active Space State Interaction (RASSI) method. Spin Hamiltonian parameters (such as $g$ factors) can be calculated from the wave functions resulting after the state interaction step employing the SINGLE_ANISO program, implemented in MOLCAS 8.0. The employed basis set has the following contractions: Dy [9s8p6d4f3g2h]; Er [9s8p6d4f3g2h]; 0 close [4s3p2d1f]; O distant [3s2p]; C [3s2p]; H [2s]. The structure of the model was extracted from the corresponding X-ray structure without any ligand simplification. Electrostatic potential maps were obtained by B3LYP calculations as implemented in the Gaussian $09_{32}$ using a TZVP basis set employing the geometry for the ligand environment of the previous CASSCF+RASSI calculations and removing the central DyIII or ErIII ion.

For the REC calculations, we used the SIMPRE computational package.33 In a first step the Radial Effective Charge (REC) model 21 was applied to the crystallographic coordinates of the first coordination sphere of DyODA and ErODA (see SI for details). The REC model is an electrostatic model that considers the effect of the coordinated atoms (X) through effective point charges located in the lanthanideligand axis at an effective distance Reff, which is equal to $r_{i}-D_{r}$, where $r_{i}$ is the distance between the atomic positions of the lanthanide and the donor atom (see fig. 1).

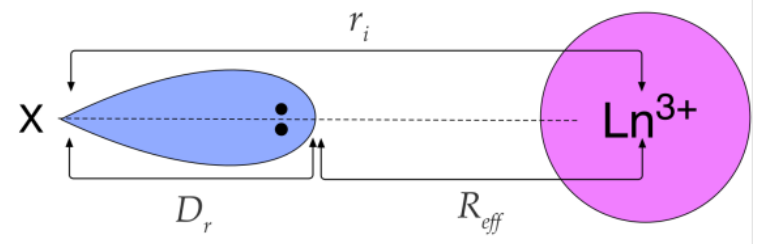

Figure 1: Electronic pair of a ligand $X$ oriented towards the nucleus of a trivalent lanthanoid cation. The effective charge is located between the lanthanoid and the donor atom $R_{e f f}=r_{i}-D_{r}$.

Oxygen donor atoms from oxydiacetate-type ligands -neither carboxylate nor ether- have not been parameterized before by the REC model. Hence, if we want to provide an inexpensive prediction of the energy levels, wave functions, $g$ components, magnetic susceptibility and magnetization of the lanthanide oxydiacetate complexes, we can take advantage of a relation that was obtained recently by fitting the experimental energy levels of the ground multiplet of the homoleptic families $\mathrm{CsNaYCl}_{6}: \mathrm{Ln}_{3}+$ and $\mathrm{CsNaYF}_{6}: \mathrm{Ln}_{3}+\mathrm{LiYF}_{4}: \mathrm{Ln}_{3}+$ and $\mathrm{LaCl}_{3}: \mathrm{Ln}_{3}+$ using the crystal structures and the REC model. 34 This approximation permits the calculation of the effective distances of the coordinated atoms using the following semiempirical formula for $D r$ :

$$
D_{r} \approx\left(\frac{N_{L}}{V_{M}}\right) \cdot \frac{1}{E_{M}\left(E_{L} \quad E_{M}\right)}
$$

where $N_{L}$ is the coordination number of the complex $\left(\mathrm{N}_{\mathrm{L}}=9\right), \mathrm{V}_{M}$ is the valence of the metal $\left(\mathrm{V}_{\mathrm{M}}=3\right)$, and Eм and EL are the Pauling electronegativities of the metal $\left(\mathrm{Eм}_{\mathrm{M}}=1.22\right.$ and $\mathrm{Eм}=1.24$ for $\mathrm{Dy}$ and $\mathrm{Er}$ respectively) and the donor atom ( $\mathrm{EL}=3.44$ for oxygen) respectively. Regarding the effective charges of the donor atoms, it could be estimated assuming the same relation $\mathrm{f}=Z_{i} \cdot D_{r}$ that the obtained between the REC parameters of two series of polyoxotungstate single-ion magnets ( $\mathrm{f}=0.094$ ), which were recently modelled and added to the general library of the model, predicting slow relaxation of the magnetization in a $\mathrm{Nd}_{3+-}$-based SIM.35 This strategy allowed us to obtain a quick estimation of the ground multiplet spectroscopic and magnetic properties in the absence of any parameter, just utilizing the previous knowledge in the study of the properties of lanthanide homoleptic coordination compounds. Thus, all the calculations presented in this manuscript are predictions, both magnetic and spectroscopic properties. For comparison, the REC model was also used via the usual procedure of fitting of the experimental data in order to add carboxylate and ether ligands to the library (details are on the SI and results concerning energy levels are represented in figs. S10-S13 and magnetic properties in figs. S14 and S15).

\section{Results and discussion}

\section{Structure}

The crystal structures of both compounds have been previously described in detail.29 Both compounds undergo spontaneous resolution on crystallisation. The central lanthanide ion coordinates three oxydiacetate anions to form a ninecoordinate complex anion, [Ln(oda)3]3-. With a coordination geometry around the lanthanide ion that can be described as distorted three-face centred trigonal prismatic, two possible helical $\Delta / \Lambda$ chiralities result for the $[\operatorname{Ln}($ oda)3]3- anion (fig. 2). 
The crystal structure can be refined in one of the Sohncke space groups (R32).

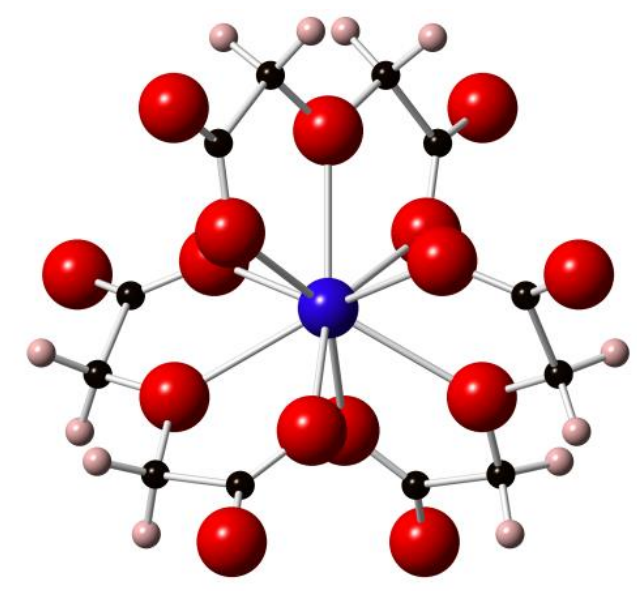

Figure 2: Vertical view of the three-blade-propeller molecular structure of $\mathrm{Na}_{5}\left[\mathrm{Dy}(\mathrm{oda})_{3}\right]\left(\mathrm{H}_{2} \mathrm{O}\right)_{6}\left(\mathrm{BF}_{4}\right)_{2}$ that emphasizes the near- $\mathrm{C} 3$ symmetry axis. The erbium derivative is isostructural.

It has been reported 29 that the formation of hydrogen bonds involving $\mathrm{BF}_{4}$ - anions could be essential in the packing of the homo-chiral complexes, resulting in the spontaneous resolution. The coordination geometry around the lanthanide ion is practically identical for both DyODA and ErODA. It may be described as slightly distorted tricapped trigonal prismatic (of $D_{3}$ symmetry), with the upper and lower triangles defined by carboxylate oxygen atoms and the capping positions (perpendicular to the nearC3 symmetry axis) occupied by ether oxygen atoms. Thus, the coordination environment is not clearly axial nor equatorial, 36 being necessary to perform structure-based theoretical calculations to know which lanthanide ion is a better candidate to exhibit SMM behaviour. 37

\section{Magnetic properties}

Dc susceptibility measurements were performed for the pure and diluted samples of both compounds $\left(Y_{(1-x)} \operatorname{Ln}(x)\right.$ with $x=0.01$ for Dy, $x=0.05$ for $\left.E r\right)$ under an applied field of $0.1 \mathrm{~T}$. The $\chi_{m} T$ values at $300 \mathrm{~K}$ are near the expected values for the $4 I 15 / 2$ and $6 H_{15 / 2}$ multiplets of Er3+ (11.27 (exp.) vs $11.48 \mathrm{emu} \cdot \mathrm{K} / \mathrm{mol}$ ) and Dy3+ (14.08 (exp.) vs $14.17 \mathrm{emu} \cdot \mathrm{K} / \mathrm{mol})$. To avoid dipolar interactions that are not included in both theoretical calculations, the experimental $\chi_{m} T$ products of the diluted samples of DyODA and ErODA are compared with the theoretical results in Fig. 3. It can be seen that the temperature-dependent magnetic susceptibility gradually decreases upon cooling due to depopulation of the electronic fine structure, reaching values close to $6 \mathrm{emu} \cdot \mathrm{K} / \mathrm{mol}$ for both complexes.

The overall shape of both experimental curves is well reproduced by all three methods (REC prediction, CASSCF and CASPT2 results). It is remarkable that the electrostatic method (blue solid line) reproduces the $\chi_{m} T$ curves with an almost excellent agreement with the experiment (red open circles) in both cases. In the case of the $a b$ initio calculated $\chi_{m} T$ product there is no improvement between CASSCF and CASPT2, being almost equivalent in the Dy derivative except at low temperature. There are noticeable differences between CASSCF (green solid line) and CASPT2 (green dashed line) in the Er example (Fig. 3 (down)), where CASPT2 seems to reproduce better the magnetic behaviour below $100 \mathrm{~K}$, while at higher temperatures CASSCF is closer to the experiment. Dynamic correlation contribution seems to be more important for the erbium system, probably for the larger electron repulsion due to the presence of two extra electrons in the $f$ orbitals in comparison with the Dy complex. Anyway, CASSCF method provides reasonable values, as expected taking into account the relatively large ionic character of the metalligand interactions. In particular, CASPT2 calculations predict a $\chi m T$ value of $11.03 \mathrm{emu} \cdot \mathrm{K} / \mathrm{mol}$, which may indicate a higher total crystal field splitting than the one obtained by the CASSCF method. Regarding the magnetization, one can observe that for the ground state the magnetic properties seem to be better reproduced using the most sophisticated methods. In both cases, the shape of the magnetization curve predicted by $a b$ initio methods coincides better with the experimental behavior, although the improvement of using CASPT2 is still not evident and generalizable: in ErODA CASPT2 results are clearly superior to those obtained using CASSCF, but the contrary is observed in DyODA. This is related with the better reproduction of the magnetic susceptibility at low temperature in the case of CASSCF (fig. 3 (up), green solid line) compared with CASPT2 (fig. 3 (down), green dashed line). On the other hand, considering the information of the magnetic susceptibility between 2 and $300 \mathrm{~K}$, the better agreement of the electrostatic calculation with the experiment allows us to think that, in these two particular examples, the energy level scheme should also be better reproduced by the semiempirical method. In the next section, we are taking advantage of spectroscopic data in order to compare both approaches with the experimental energy level schemes for these two complexes and elucidate this question (see figures 5 and 6). 

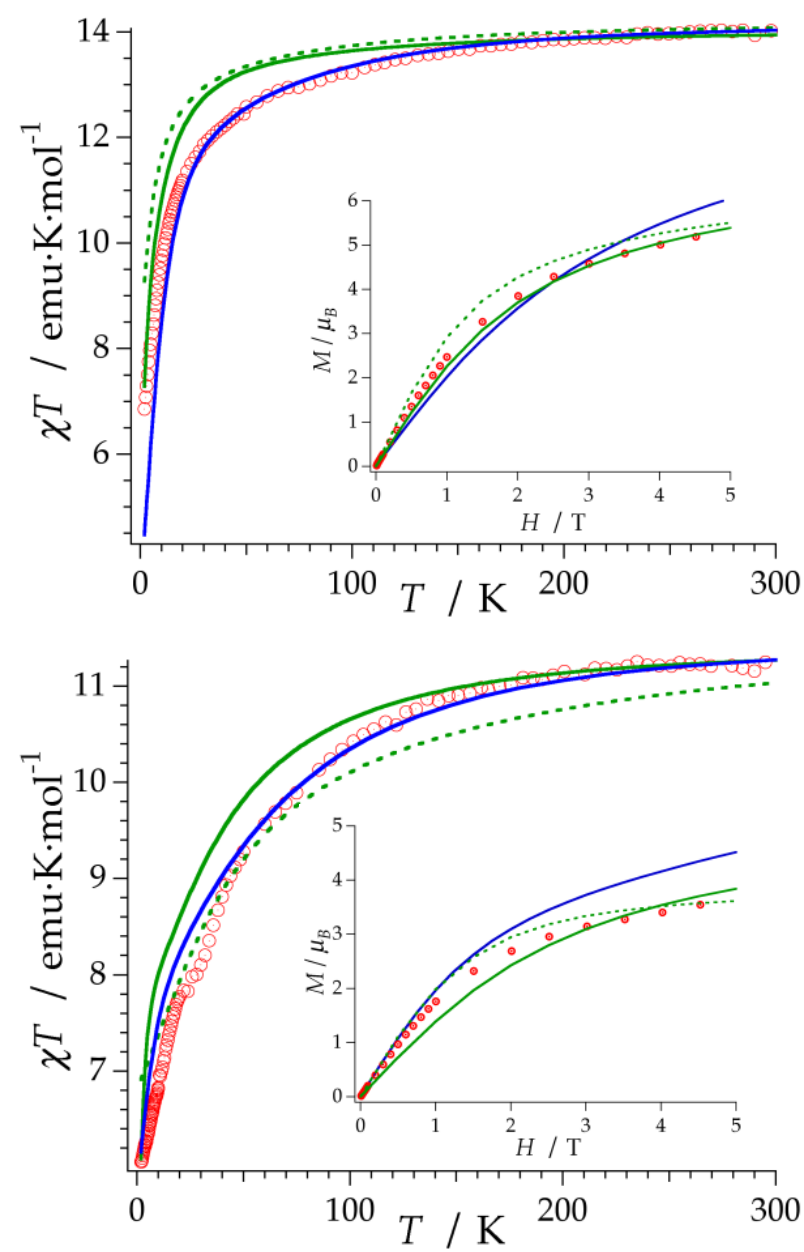

Figure 3: Comparison of the $\chi_{m} T$ product for the magnetically diluted powder samples of $\mathrm{Y}_{0.99} \mathrm{Dy} 0.01 \mathrm{ODA}$ (up) and $\mathrm{Y}_{0.95} \mathrm{Er}_{0.05 \mathrm{ODA}}$ (down) at $\mathrm{H}$ $=1000$ Oe. Inset: Magnetization at $5 \mathrm{~K}$ of $Y_{0.99 D y 0.01}$ ODA (up) and Y0.95Er0.05ODA (down). Red open circles: exp.; blue solid line: REC prediction, green solid line: CASSCF, green dashed line: CASPT2.

Ac susceptibility measurements were performed for both compounds above $2 \mathrm{~K}$. In both cases, they reveal the typical features associated with the SMM behaviour for a system with some mixture of $M_{J}$ components enabling the possibility of presenting avoided hyperfine crossings and quantum tunneling. Hence, in the absence of a dc field there is a weak frequency-dependent signal in $\chi^{\prime \prime}$ but no clear $\chi^{\prime}$ signal (fig. S5(left)). The system is taken beyond the hyperfine crossing region after applying an external field of 1000 Oe. As a result, both $\chi^{\prime}$ and $\chi^{\prime \prime}$ show strong frequency dependencies. This indicates the presence of a slow relaxation process involving an energy barrier for the reversal of the magnetization. In the Dy derivative, a maximum is detected between 2.5 and $3.0 \mathrm{~K}$ in $\chi^{\prime}$ (Fig. 4 (top, left)), which is frequency dependent. In $\chi^{\prime \prime}$, the maximum could not be observed in the measurements carried out between 2 and $10 \mathrm{~K}$ (Fig. 4 (down, left)). The upward trend from $4 \mathrm{~K}$ to $2 \mathrm{~K}$ permits to expect that the position of the maximum should appear between 1 and $2 \mathrm{~K}$, but this is outside our operating range. On the other hand, the magnetic properties of ErODA reveal the typical features associated with SMM behaviour. Thus, both $\chi^{\prime}$ and $\chi^{\prime \prime}$ under an applied magnetic field of 1000 Oe (Fig. 4 (right)) show strong frequency dependencies, which indicates the presence of a slow relaxation process involving an energy barrier for the reversal of the magnetization. Depending on the frequency of the applied ac field, $\chi^{\prime}$ presents a maximum between 4.3 and $4.9 \mathrm{~K}$, while $\chi^{\prime \prime}$ has also a maximum between 3.5 and $4.2 \mathrm{~K}$ for 1500 and $10000 \mathrm{~Hz}$, respectively (Fig. 4 (right, down)). Dynamic susceptibility measurements under an alternating field for the diluted ErODA compound are presented in fig. S6. An Arrhenius analysis was performed, which, as reported in the SI (fig. S7), results in an effective barrier of $31 \mathrm{~cm}-1$. As discussed in the next section, spectroscopic information allows us to discard this effective barrier as an artifact.
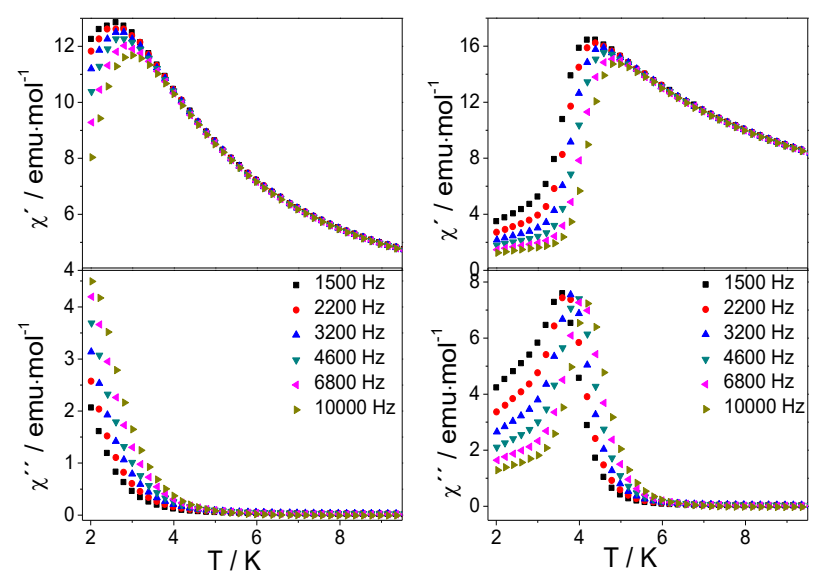

Figure 4: In-phase (top) and out-of-phase (down) dynamic susceptibility of DyODA (left) and ErODA (right) under an applied dc field of $1000 \mathrm{Oe}$ at the frequencies shown in the legend.

Theoretical analysis using the energy level scheme

In references 25 and 26, a large number of energy levels were located and assigned for both members of the LnODA family (65 for Er, 152 for Dy) by a combination of spectroscopic techniques, which in both studies consisted of optical absorption (unpolarized, linearly and circularly polarized), and was also extended to optical emission (also including all polarizations) for DyODA. Fits assumed a trigonal CF symmetry, thus in each case six CF parameters ( $B_{2}^{0}, B_{4}^{0}, B_{6}^{0}, B_{4}^{3}, B_{6}^{3}$ and $B_{6}^{6}$ ) were varied in order to find a complete description of the experimental properties. In both derivatives, the root-mean-square deviation between the fit and the experimental data is below $10 \mathrm{~cm}-1$ for the whole spectrum, and below 7 $\mathrm{cm}-1$ for the ground $J$ manifold, so this is the expected accuracy for the missing experimental levels in the case of the erbium derivative. Note that this accuracy is maintained for energies in the tens of thousands of $\mathrm{cm}-1$, accounting for the power of this approach and 
justifying its use as benchmark or "gold standard" to judge the quality of theoretical calculations when not all the experimental energy levels are available. The phenomenological CFPs extracted from the fit in refs. 27 and 28 are reported together with the predicted ones by CASSCF and the REC model in ESI. The wave functions of the ground state and the input coordinates used in both calculations are also included.

In figures 5 (DyODA) and 6 (ErODA), we compare these reference data with different sets of theoretical energy levels using the real structures of both complexes, by three different strategies. We have to stress that, in this case, the application of the REC model does not rely on any free fitting parameters, as we explained in the experimental section.

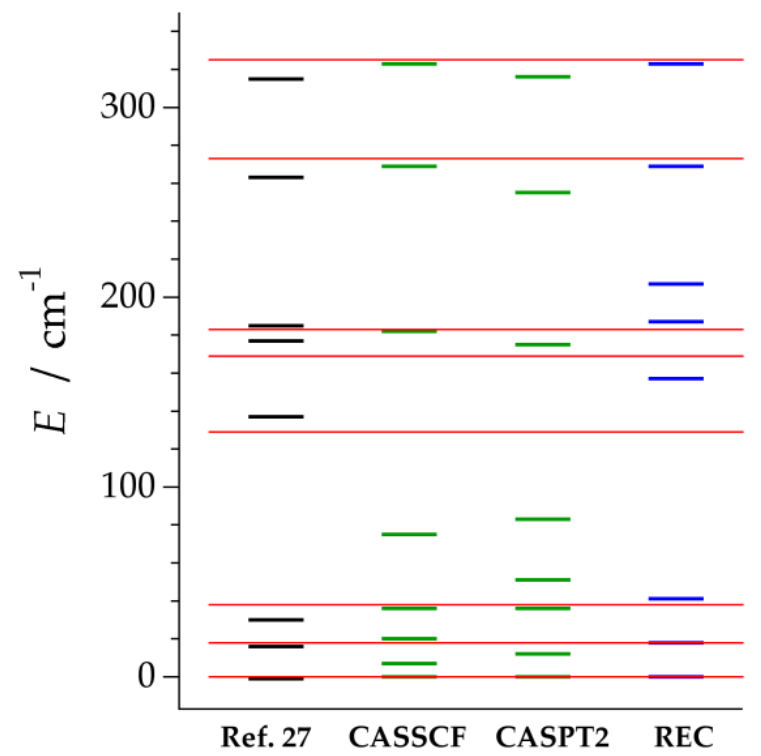

Figure 5: Crystal field energy level scheme of the ground $J$ multiplet of DyODA. Thin red lines: experimental optical spectrum. Thick lines: spectroscopic fit (Ref. 27), CASSCF/CASPT2 energies including spinorbit effects and REC model prediction, as indicated at the axis (details in the text).

The experimental energy level scheme of DyODA can be described as a bunching of levels in three groups $3+3+2$, where each group has a width of about $50 \mathrm{~cm}-1$ and there is a gap with no levels of about $80 \mathrm{~cm}-1$ between every two groups. This $3+3+2$ description is well reproduced by the semiempirical REC prediction. In contrast, both CASSCF and CASPT2 respond rather to a $5+1+2$ scheme, that is, the fourth and fifth energy levels, that experimentally are found near $150 \mathrm{~cm}-1$, are predicted to be about a $50 \%$ lower in energy, around $75 \mathrm{~cm}-1$. The total energy level splitting, of about $320 \mathrm{~cm}-1$, is adequately reproduced by all methods. The CASPT2 method improves CASSCF results for the low lying state energies, that are crucial for the theoretical determination of the anisotropy barriers. On the other hand, the prediction provided by the REC model using equation
1 offers a remarkable reproduction of the scheme, as confirmed in the correct prediction of the $\chi_{m} T$ product. In addition, one can observe that following this methodology the total CF splitting is perfectly determined and the scheme $3+3+2$ is also obtained. This is not trivial, as different values of Reff will modify the ratio between $2 \mathrm{nd}-$, 4 th- and 6th-rank parameters, changing the levels distribution. In this particular case, this predictive electrostatic approach results in a very satisfactory description of the energy levels.

For the Er derivative, five of the eight CF Kramers doublets split out of the $4 I 15 / 2$ (ground) multiplet were reported.28 Taking the spectroscopic fit as a reference for the whole set of levels (black thick line in fig. 6), a slightly different $2+3+3$ bunching is observed, with bunches that are at the same time increasingly wider $(40,70$ and $110 \mathrm{~cm}-1)$ and more separated than in the Dy case (over $100 \mathrm{~cm}-1$ for each of the inter-bunch separation).

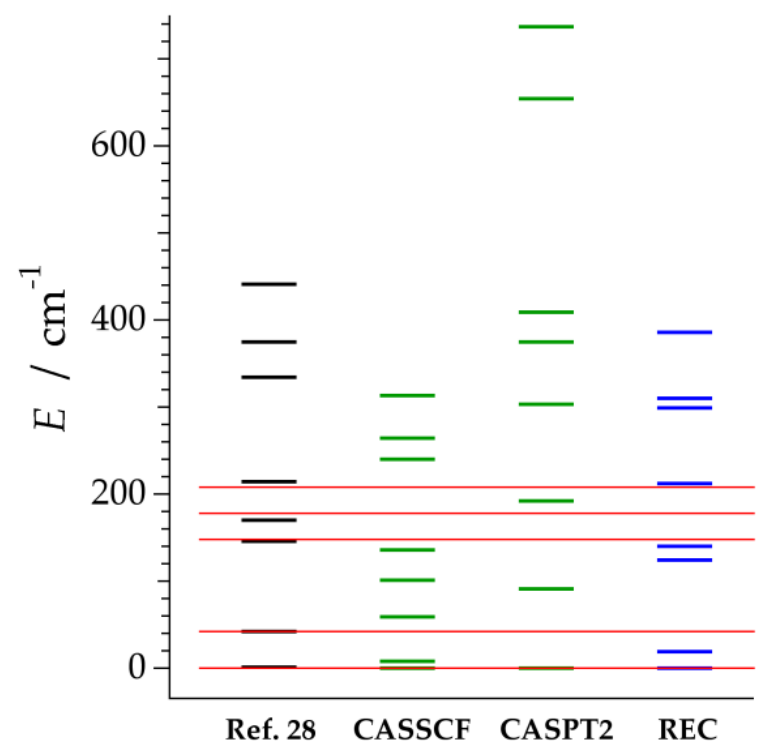

Figure 6: Crystal field energy level scheme of the ground $J$ multiplet of ErODA. Thin red lines: experimental optical spectrum. Thick lines: fit (Ref. 28), CASSCF/CASPT2 energies including spin-orbit effects and REC model prediction, as indicated at the axis (details in the text).

In this case, the CASSCF calculation qualitatively predicts the $2+3+3$ bunches, but underestimates all energies, so that in general the CASSCF levels are situated in the energies between the experimentally found energy bunches. Interestingly, while in this case an overall increase in CASSCF energies of about $50 \%$ that would improve the agreement, the factor would be of the order of $400 \%$ if only the first excited state is considered, highlighting the risk of using these factors with partial information, as it was done in references 10 and 26. As reflected in the $\chi_{m} T$ curve (Fig. 3 (down)) in the case of CASPT2 the difference between the calculated energy levels with respect to CASSCF is striking, but also pretty far from 
the experimental result with no clear improvement. In this case, a $1+6+2$ bunching is found with a total splitting of about $740 \mathrm{~cm}-1$, which is a $68 \%$ higher than the results of the phenomenological fit $(441 \mathrm{~cm}$ 1). Such a total splitting seems unreasonable and is key to understand the deviation of the $\chi_{m} T$ product respect to the experiment (see Fig. 3 (down), green dashed line). On the other hand, the REC prediction is not as accurate as the one calculated in DyODA, but still respects the $2+3+2$ scheme. The total splitting calculated by the REC model is very close to the one calculated with the phenomenological fit and thus is expected to be close to the actual energy levels.

As mentioned in the previous section, the magnetic data suggest an Orbach mechanism, but in this case we can positively discard that a relaxation via an excited state happens at $31 \mathrm{~cm}-1$, since the spectroscopy determines the first excited state to be at $40 \mathrm{~cm}-1$ (fig. 6). It is important to note that it has been very common in this field to assume an Orbach process without proof and without spectroscopic information to back it up. Furthermore, by inspecting the typical errors of CASSCF, CASPT2 and the REC model, it is easy to see that sometimes the experimental effective barrier will, by mere chance, be in the range of a theoretical prediction, giving an appearance of confirmation. Moreover, while in some cases the Arrhenius plot displays some curvature, pointing to other relaxation pathways (Raman, quantum tunneling or a direct process), no signs of this can be found in the case of ErODA. It is to be expected that in a number of cases an Orbach mechanism has been mistakenly proposed, either by default or even with an appearance of theoretical confirmation, and only spectroscopic information will eventually allow to correct these errors.

\section{Limitations of CASSCF, CASPT2 and the REC model}

After analysing these two particular examples, let us critically review general limitations of $a b$ initio calculations and the electrostatic approach. For clarity, let us first briefly enumerate the limitations of each approach, and then go into some detail. In this regard, Complete Active Space ab initio calculations:

(1) consider a single complex (the results should be compared with those obtained with magnetically dilute samples),

(2) apply perturbations in the wrong order, in CASPT2 both dynamic correlation and spin-orbit effect are included perturbatively.

(3) are limited by large computacional requirements.

Even when more than a single metal is considered, dipolar interactions within the crystals are also outside the scope of this approach. More crucially, the fact that the SINGLE_ANISO procedure applies spin-orbit coupling after, rather than before, the ligand field, has fundamental, limiting consequences in the accuracy of the method that have not yet been adequately evaluated. On top of that, there are the non-fundamental limits posed by large computacional requirements, especially in the case of the CASPT2 method. Because it is a computationally demanding method, it can happen that the end results have not converged, that is, that using a larger basis set, active space or contraction would produce results that are closer to the experimental data. Again, an extra theoretical effort will eventually overcome (2), but it will require programming new calculation procedures. However, electronic structure methods provide several useful pieces of information $(g$ components, energy of the states, probability of the different spin relaxation mechanism between states and so on) and tools for the understanding of the magnetic properties (electrostatic potential of the ligands, shape of the electron density).

The semiempirical REC model considers:

(1) a single metal,

(2) the first coordination sphere

(3) the ground $J$ multiplet

(4) it is a parametric (semiempirical) method that often relies on low-quality data (powder $\chi_{m} T$ ) data and assumes parameters are re-usable.

As it considers a single metal, this method is inappropriate for cluster-type complexes. Limiting the point charges to the first coordination sphere can have severe consequences for the prediction of the easy axis of magnetization, while limiting the treatment to the ground $J$ means the upper levels, even within the ground $J$, are less well described, and their energies are often overestimated. Being semiempirical means there is no systematic method to obtain more accurate CF parameters, other than fitting higher-quality (spectroscopic) experimental data. That includes the risk of assuming that parameters extracted from a compound can be used on a different one. Some of these points can be improved by some extra theoretical effort. For example (3), is solved by considering the full singleion Hamiltonian (e.g. using the REC parameters in the CONDON computational package), 14 while (4) is continuously being improved as the number of examples studied by this method grow, which provide a better understanding of the adequate parameterization of each kind of ligand. 36

Finally, as expected, the spectroscopic fit offers a perfect description of the measured levels. Nevertheless, it is important to point out that such a phenomenological approach can only be carried out after the energy level scheme is properly determined and it is only adequate when the symmetry of coordination environment is comparable to a point group, thus reducing the number of $\mathrm{CF}$ parameters. 
Most of the coordination complexes of interest in molecular magnetism present coordination environments that provide 27 non-negligible crystal field parameters, and thus require models based on the prediction of the properties from the real structure, such as the two approaches that have been compared and discussed here.

\section{Concluding remarks}

In this work we have successfully used spectroscopic information of previous works to anticipate the single-molecule behaviour of the dysprosium and erbium derivatives of an oxydiacetate complex series. Their magnetic properties under ac and dc fields were experimentally determined, and for their study we performed different kinds of state-of-the-art theoretical calculations to evaluate and compare their predicting capabilities. Taking the magnetic measurements performed in this work together with spectroscopic transition data as a reference, one needs to conclude that current Complete Active Space $a b$ initio methods can offer a reasonable reproduction of the magnetic properties of both compounds, but fail to account for the energy level distribution, including the energy of the first excited state. In this sense, there is no clear benefit for all cases in the extra computational cost of using more sophisticated models, i.e. for these tasks CASPT2 does not prove to be superior to CASSCF nor does CASSCF prove to be superior over REC. In these two examples, the simple electrostatic REC model offers an inexpensive tool to get a promising initial prediction of CFPs, energy levels and magnetic properties. Note, however, that this is a rather favourable case for REC model, with a nearhomoleptic coordination sphere which enhances the predictive character of the REC mode.

\section{ASSOCIATED CONTENT}

Details about the experimental measurements and the theoretical calculations are provided as supporting information.

\section{AUTHOR INFORMATION}

\section{Corresponding Author}

*Email: eugenio.coronado@uv.es

*Email: eliseo.ruiz@qi.ub.es

\section{Author Contributions}

The manuscript was written through contributions of all authors. / All authors have given approval to the final version of the manuscript.

\section{Funding Sources}

EU, Spanish MINECO, Generalitat Valenciana and Generalitat de Catalunya.

\section{ACKNOWLEDGMENT}

The present work has been funded by the EU (ERC Advanced Grant SPINMOL and ERC Consolidator Grant DECRESIM), the Spanish MINECO (MAT201456143-R, CTQ2014-52758-P, CTQ2011-23861-C0201 and 'Unidad de Excelencia María de Maeztu' MDM-2015-0538 granted to ICMol), and the Generalitat Valenciana (Prometeo and ISIC Programmes of excellence). A.G.-A. acknowledges funding by the MINECO (Ramón y Cajal contract). J.J.B. thanks the Spanish MECD for an FPU predoctoral grant. We also acknowledge J. M. Martínez-Agudo (University of Valencia) for his help with the magnetic measurements. E.R. thanks the Generalitat de Catalunya for an ICREA Academia grant. We thank computer time and facilities provided by Consorci de Serveis Universitaris de Catalunya (CSUC).

\section{REFERENCES}

1 R. Giraud, W. Wernsdorfer, A. M. Tkachuk, D. Mailly and B. Barbara, Phys. Rev. Lett., 2001, 87, 057203.

2 N. Ishikawa, M. Sugita, T. Ishikawa, S. Koshihara and Y. Kaizu, J. Am. Chem. Soc., 2003, 125, 8694-8695.

3 M. A. AlDamen, J. M. Clemente-Juan, E. Coronado, C. MartiGastaldo and A. Gaita-Ariño, J. Am. Chem. Soc., 2008, 130, 8874-8875.

4 S.-D. Jiang, B.-W. Wang, G. Su, Z.-M. Wang and S. Gao, Angew. Chem.-Int. Ed., 2010, 49, 7448-7451.

5 D. N. Woodruff, R. E. P. Winpenny and R. A. Layfield, Chem. Rev., 2013, 113, 5110-5148.

6 D. Gatteschi and R. Sessoli, Angew. Chem.-Int. Ed., 2003, 42, 268-297.

7 F. Troiani and M. Affronte, Chem. Soc. Rev., 2011, 40, 3119 3129.

8 R. Sessoli, D. Gatteschi, A. Caneschi and M. A. Novak, Nature, 1993, 365, 141-143.

9 W.T. Carnall, P.R. Fields and B.G. Wybourne, J. Chem. Phys. 1965, 42, 3797.

10 R. Marx, F. Moro, M. Dörfel, L. Ungur, M. Waters, S. D. Jiang, M. Orlita, J. Taylor, W. Frey, L. F. Chibotaru and J. van Slageren, Chem. Sci., 2014, 5, 3287-3293.

11 K. S. Pedersen, L. Ungur, M. Sigrist, A. Sundt, M. SchauMagnussen, V. Vieru, H. Mutka, S. Rols, H. Weihe, O. Waldmann, L. F. Chibotaru, J. Bendix and J. Dreiser, Chem. Sci., 2014, 5, 1650-1660.

12 G. Cucinotta, M. Perfetti, J. Luzon, M. Etienne, P. E. Car, A. Caneschi, G. Calvez, K. Bernot and R. Sessoli, Angew. Chem.Int. Ed., 2012, 51, 1606-1610.

13 J. Luzón and R. Sessoli, Dalton T., 2012, 41, 13556-13567.

14 H. Schilder and H. Lueken, J. Magn. Magn. Mater. 2004, 281, 17-26.

15 J. van Leusen, M. Speldrich, H. Schilder and P. Kögerler, Coord. Chem. Rev. 2014, DOI:10.1016/j.ccr.2014.10.011.

16 H. Bethe, Ann. Phys.-Berlin, 1929, 3, 133-208.

17 P. Porcher, M. C. Dos Santos and O. Malta, Phys. Chem. Chem. Phys., 1999, 1, 397-405.

18 W. Urland, Chem. Phys., 1976, 14, 393-401.

${ }_{19}$ C. K. Jorgensen, R. Pappalardo and H. H. Schmidtke, J. Chem. Phys., 1963, 39, 1422-1430.

20 O. L. Malta, Chem. Phys. Lett., 1982, 87, 27-29. 
21 O. L. Malta, Chem. Phys. Lett., 1982, 88, 353-356.

22 J. J. Baldoví, J. J. Borrás-Almenar, J. M. Clemente-Juan, E. Coronado and A. Gaita-Ariño, Dalton T., 2012, 41, 1370513710.

23 J. Ruiz, A.J. Mota, A. Rodríguez-Diéguez, S. Titos, J.M. Herrera, E. Ruiz, E. Cremades, J.P. Costes, E. Colacio, Chem. Commun. 2012, 48, 7916-7918.

24 D. Aravena and E. Ruiz, Inorg. Chem., 2013, 52, 13770 13778.

25 S. Gómez-Coca, D. Aravena, R. Morales, E. Ruiz, Coord. Chem. Rev., 2015, 289, 379-392.

26 E. Moreno Pineda, N. F. Chilton, R. Marx, M. Dörfel, D. O. Sells, P. Neugebauer, S.-D. Jiang, D. Collison, J. van Slageren, E. J. L. McInnes and R. E. P. Winpenny, Nat. Comm., 2014, 5, 5243.

27 D.H. Metcalf, T.A. Hopkins, F.S. Richardson, Inorg. Chem. 1995, 34, 4868-4878.

28 K.A. Schoene, J.R. Quagliano, F.S. Richardson, Inorg. Chem. 1991, 30, 3803-3812

29 A. Lennartson and M. Hakansson, CrystEngComm, 2009, 11, 1979-1986.

30 L. F. Chibotaru and L. Ungur, J. Chem Phys, 2012, 137, 064112.

31 Aquilante, F., De Vico, L., Ferré, N., Ghigo, G., Malmqvist, P.Å., Neogrády, P., Pedersen, T. B., Pitoňák, M., Reiher, M., Roos, B. O., Serrano-Andrés, L., Urban, M., Veryazov, V. and Lindh, R., J. Comput. Chem., 31, 224 (2010).

32 Frisch, M. J.; Trucks, G. W.; Schlegel, H. B.; Scuseria, G. E.; Robb, M. A.; Cheeseman, J. R.; Scalmani, G.; Barone, V.; Mennucci, B.; Petersson, G. A.; Nakatsuji, H.; Caricato, M.; Li, X.; Hratchian, H. P.; Izmaylov, A. F.; Bloino, J.; Zheng, G.; Sonnenberg, J. L.; Hada, M.; Ehara, M.; Toyota, K.; Fukuda, R.;
Hasegawa, J.; Ishida, M.; Nakajima, T.; Honda, Y.; Kitao, 0.; Nakai, H.; Vreven, T.; Montgomery, J., J. A.; Peralta, J. E.; Ogliaro, F.; Bearpark, M.; Heyd, J. J.; Brothers, E.; Kudin, K. N.; Staroverov, V. N.; Kobayashi, R.; Normand, J.; Raghavachari, K.; Rendell, A.; Burant, J. C.; Iyengar, S. S.; Tomasi, J.; Cossi, M.; Rega, N.; Millam, N. J.; Klene, M.; Knox, J. E.; Cross, J. B.; Bakken, V.; Adamo, C.; Jaramillo, J.; Gomperts, R.; Stratmann, R. E.; Yazyev, O.; Austin, A. J.; Cammi, R.; Pomelli, C.; Ochterski, J. W.; Martin, R. L.; Morokuma, K.; Zakrzewski, V. G.; Voth, G. A.; Salvador, P.; Dannenberg, J. J.; Dapprich, S.; Daniels, A. D.; Farkas, Ö.; Foresman, J. B.; Ortiz, J. V.; Cioslowski, J.; Fox, D. J. Gaussian09; Gaussian: Wallingford, CT, 2009.

33 (a) J. J. Baldovi, S. Cardona-Serra, J. M. Clemente-Juan, E. Coronado, A. Gaita-Ariño and A. Palii, J Comput Chem, 2013, 34, 1961-1967. (b) J. J. Baldovi, J. M. Clemente-Juan, E. Coronado, A. Gaita-Ariño and A. Palii, J Comput Chem, 2014, 35, 1930-1934.

34 J.J. Baldoví, E. Coronado and A. Gaita-Ariño, Dalton Trans. 2015, 44, 12535-12538.

35 J.J. Baldoví, J.M. Clemente-Juan, E. Coronado, Y. Duan, A. Gaita-Ariño and C. Giménez-Saiz, Inorg. Chem., 2014, 53, 9976-9980.

36 J.D. Rinehart and J.R. Long, Chem. Sci., 2011, 2, 2078-2085.

37 J.J. Baldoví, S. Cardona-Serra, J.M. Clemente-Juan, E. Coronado, A. Gaita-Ariño and A. Palii, Inorg. Chem., 2012, 51, 12565-12574.

38 A. D. Becke, J. Chem. Phys. 1993, 98, 5648-5652

39 A. Schaefer, C. Huber, R. Ahlrichs, J. Chem. Phys. 1994, 100, 5829-5835. 